\title{
Understanding Atomic-Scale Phenomena in Functional Materials by using STEM, ELNES, and Theoretical Calculations
}

\author{
${ }^{1}$ Teruyasu Mizoguchi, ${ }^{2}$ Tetsuya Tohei, ${ }^{2,3}$ Yukio Sato, ${ }^{2,3}$ Takahisa Yamamoto, ${ }^{2,3,4}$ Yuichi Ikuhara, \\ ${ }^{5}$ Hiromichi Ohta, ${ }^{6}$ Hidenori Hiramatsu, and ${ }^{6,7}$ Hideo Hosono \\ ${ }^{1}$ Institute of Industrial Science, The University of Tokyo, Tokyo, Japan \\ ${ }^{2}$ Institute of Engineering Innovation, The University of Tokyo, Tokyo, Japan \\ ${ }^{3}$ NSRL, Japan fine ceramics center, Nagoya, Japan \\ ${ }^{4}$ WPI-AMRI, Tohoku University, Sendai, Japan \\ ${ }^{5}$ Graduate School of Engineering, Nagoya University, Nagoya, Japan \\ ${ }^{6}$ ERATO-SORT, JST, Frontier Research Center, Tokyo Institute of Technology, Yokohama, Japan \\ ${ }^{7}$ Frontier Research Center, Tokyo Institute of Technology, Yokohama, Japan
}

In order to understand diffusions, segregations, and doping behaviors in functional materials, STEM, ELNES, and theoretical calculations were performed. Following topics will be mainly presented; 1) visualization of a dopant in newly discovered superconductor, 2) diffusions in $\mathrm{SrTiO}_{3}$ based superlattices, and 3) dopants segregations in $\mathrm{ZnO}$ grain boundary.

1) $\mathrm{LaFeOAs}$ is newly discovered superconductor, and it is known that the pure LaFeOAs does not exhibit superconductivity in the stoichiometric composition [1]. Instead, the undoped materials undergo a structural phase transition and antiferromagnetic ordering at low temperature. Doping electrons or holes into the system suppresses the structural transition and the magnetic order, and superconductivity emerges above a critical doping concentration. Such carrier doping is achieved by the partial substitution of the constituent ions by aliovalent ions: Fluorine is typical in the case of LaFeOAs. However, so far no microscopic observations have been made on the dopant state in these systems because the fluorine is believed to be substituted for the oxygen sites and is invisible to conventional imaging techniques. We investigated the fluorine doped LaFeOAs by using HAADF-STEM and EELS spectrum imaging technique. Figure 1(b) shows HAADF-STEM image of fluorine doped LaFeOAs. Although La, Fe, and As are distinguished in the HAADF-STEM image, oxygen and fluorine are invisible in the image. Figures 1(c)-(f) shows the results of the spectrum imaging for $\mathrm{La}-\mathrm{M}_{4,5}, \mathrm{Fe}-\mathrm{L}_{2,3}$, and F-K edges. It is seen that the spatial distribution of fluorine ions, which is a key for the superconductivity but is invisible by HAADF imaging, is clearly visualized at the oxygen layer [2].

2) $\mathrm{SrTiO}_{3} / \mathrm{SrTi}_{0.8} \mathrm{Nb}_{0.2} \mathrm{O}_{3} / \mathrm{SrTiO}_{3}$ superlattice shows a giant thermoelectric property because of the two-dimensional electron gas formed at the Nb-doped layer [3]. In this study, diffusion behaviors in $\mathrm{SrTiO}_{3} / \mathrm{SrTi}_{0.8} \mathrm{Nb}_{0.2} \mathrm{O}_{3} / \mathrm{SrTiO}_{3}$ and $\mathrm{BaTiO}_{3} / \mathrm{SrTi}_{0.8} \mathrm{Nb}_{0.2} \mathrm{O}_{3} / \mathrm{SrTiO}_{3}$ superlattices were investigated by using STEM-ELNES and first principles calculations. It was found that the diffusion behaviors of $\mathrm{Nb}$ and $\mathrm{Ba}$ are completely different, and strongly influence to the electric properties.

3) Lanthanide dopants in $\mathrm{ZnO}$ grain boundary play key role to bring about non-linear 
current-voltage characteristics [4]. Thus, the understanding of the segregation behaviors of those lanthanide dopants is essential for further developing and controlling the materials properties. We investigated the segregation behavior of $\mathrm{Ce}$ and $\mathrm{Eu}$ at the $\mathrm{ZnO}$ grain boundaries by STEM, EELS, and theoretical calculations, and revealed the dominant factor for the dopant segregations.

The details of those topics will be presented and discussed [5].

[1] Y. Kamihara, T. Watanabe, M. Hirano, and H. Hosono, J. Am. Chem. Soc., 130, 3296 (2008).

[2] T. Tohei, T. Mizoguchi, H. Hiramatsu, Y. Kamihara, H. Hosono, and Y. Ikuhara, Appl. Phys. Lett. 95 193107-1-3 (2009).

[3] H. Ohta, S.W. Kim, Y. Mune, T. Mizoguchi, K. Nomura, S. Ohta, T. Nomura, Y. Nakanishi, Y. Ikuhara, M. Hirano, H. Hosono, K. Koumoto, Nature Materials, 6, (2007) 129-134.

[4] Y. Sato, J. P. Buban, T. Mizoguchi, N. Shibata, M. Yodogawa, T. Yamamoto, and Y. Ikuhara, Phys. Rev. Lett. 97 (2006) 106802-1-4.

[5] K. Koumoto, N. Shibata, S. Hidaka, S. Obayashi, Y. Mune, and N. Saito are acknowledged for their helpful supports. A part of this study was supported by Grant-in-Aid for Scientific Research on Priority Areas "Nano Materials Science for Atomic Scale Modification 474" from Ministry of Education, Culture, Sports, Scienceand Technology (MEXT) of Japan.

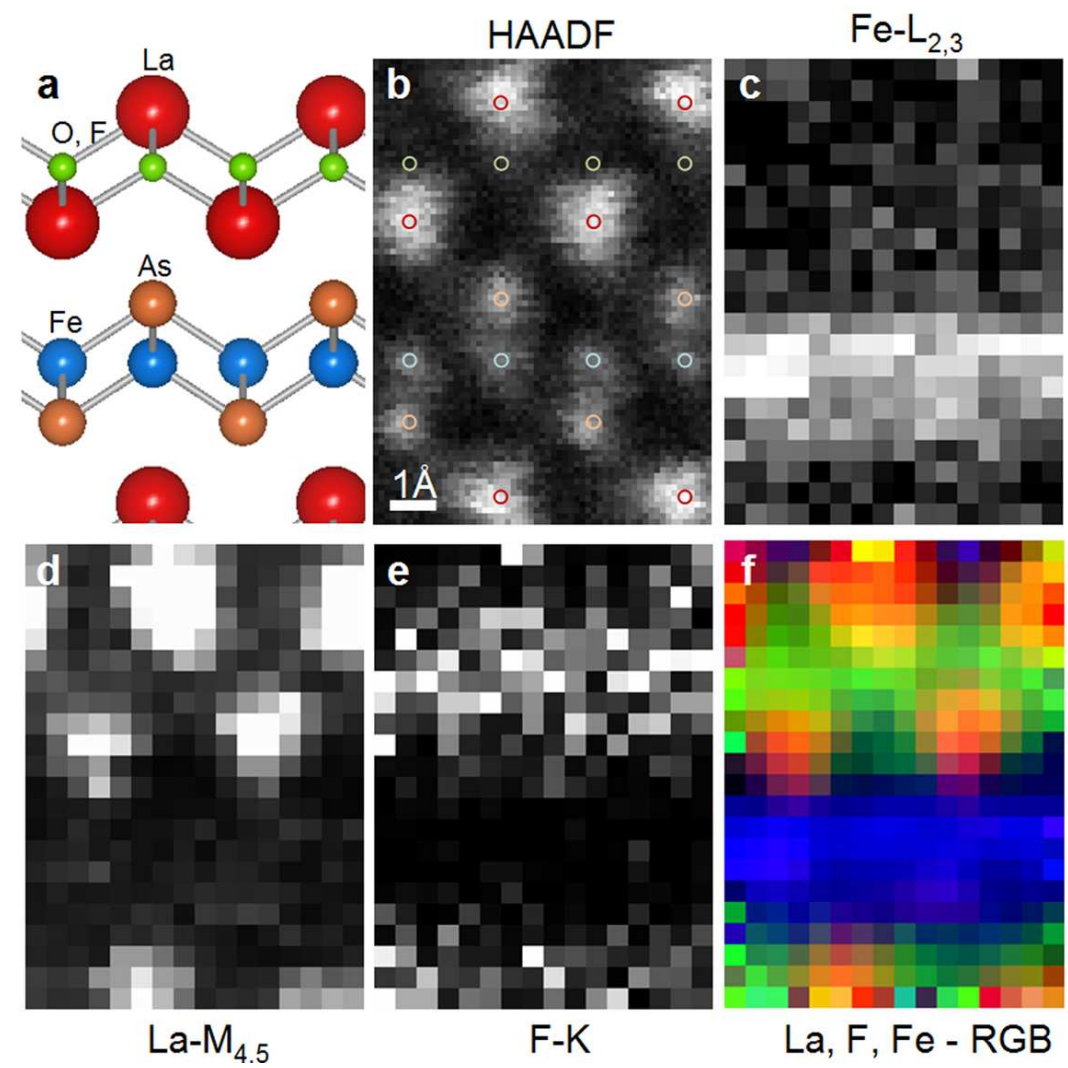

Fig. 1 (a) atomic structure of LaFeOAs along [100] direction, (b) HAADF-STEM image, and (c)-(e) spectrum imaging of $\mathrm{Fe}-\mathrm{L}_{2,3}, \mathrm{La}_{\mathrm{M}} \mathrm{M}_{4,5}$, and F-K edges, and (f) superimposed image with RGB colors. 